\title{
SIMULATION Wi-FI NETWORK WITH WIRELESS DISTRIBUTION SYSTEM (WDS) TOPOLOGY
}

\author{
Rohmat Tulloh \\ School Of Applied Science Telkom University Bandung, Indonesia \\ rohmatth@telkomuniversity.ac.id \\ Hasanah Putri \\ School Of Applied Science Telkom University Bandung, Indonesia \\ hasanahputri@tass.telkomuniversity.ac.id \\ Dwi Andi Nurmantris \\ School Of Applied Science Telkom University Bandung, Indonesia \\ dwiandi@tass.telkomuniversity.ac.id \\ Desi Dwi Prihatin \\ School Of Applied Science Telkom University Bandung, Indonesia \\ desidwip18@gmail.com
}

\begin{abstract}
At present, the need for internet access in public area using wireless connections is increasing drastically and this should be supported by good network infrastructure. Wireless network is one of the best alternative in building a practical internet network. WDS is a system to develop wireless internet network without having to use cable as its backbone for access point but instead utilize wireless path from its access point. The problems that often arise in the design of wireless LAN network is the poor signal received by the user due to the installation of an access point that is poor location-wise. This is because at some point it is very difficult to install Access point using cable because the distance is too far from the existing router. In this paper, we propose to use three scenario of WDS mode to compare its performance. We investigate three different topology policy models of the WDS Bridge point-to-point, WDS Bridge point to multipoint and WDS repeater. The use of these three topologies aims to reach locations that are not covered by wireless LAN network, in addition this method can save the use of network resources such as LAN cables. Based on the simulation results, it is found that the topology point to point gives better value but it generated higher delays. The delay generated on point to point is higher than other topologies. Meanwhile, the measurement results are not much different on topology point to multipoint with repeater topology. The point to multipoint topology is slightly better than the repeater of the three QoS parameters.
\end{abstract}

\section{Indexing terms/Keywords}

WDS, Wireless LAN, Repeater, QoS, Access Point

\section{Academic Discipline And Sub-Disciplines}

Computer Communication; Internet

\section{SUBJECT CLASSIFICATION}

System Design

\section{TYPE (METHOD/APPROACH)}

\author{
Quasi-Experimental
}

\section{INTRODUCTION}

In recent years we have witnessed a very large spread in the application of WLAN technology based on the IEEE802.11 standard [1] [2]. In telecommunication networks, "bridges" are products that connect local area networks (LANs) to other local area networks that use the same protocol (eg Ethernet or token ring). You can imagine a "bridge" as a device that determines whether a message from you to another person will go to a local area network in your building or to someone in a local area network in a building across the street. A "bridge" checks every message on a LAN, "conveys" it is known to be on the same LAN, and passes on that is known to be on another path. Interconnected LAN (or LAN set), you can build a wireless network with a large wireless network using Wireless Distribution System (WDS). WDS technology allows access points to communicate with each other wirelessly by default. WDS technology requires the use of MAC (Media Access Control) to establish relationships within WDS groups. It brings connectivity to the data link layer of the OSI model, which is a layer under traditional IP-based connectivity [7].

However, these technologies still experience certain inefficiencies such as: low throughput, delay and high jitter. This technology is not recommended for real time services such as peer-to-peer and interactive applications that are commonly used such as E-learning, VolP, VoD, etc. Recent researches attempts to propose enhancements to find solutions[3]. Although this technology has the deficiencies described earlier, it is commonly used and proposed as an alternative to improve spectrum electromagnetic occupancy as each client is connected to its own Access Point (AP) rather than to the exterior node [4]. With WDS, AP can communicate with each other with wireless links. Therefore, without the need for expensive Ethernet cable installations, WDS can easily increase wireless coverage. [5] 


\section{METHODS}

A. Wireless Distribution System (WDS)

In the IEEE 802.11 standard, there exist two operation modes: ad-hoc mode and infrastructure mode. In the infrastructure mode, one AP and its associated stations form a basic service set (BSS). Several BSSs can be connected with each other by distribution system (DS), which forms an extended service set (ESS). Within an ESS, mobile stations roam and stay connected to the available network resource. DS is usually wired link and a typical example of DS is the Ethernet. DS can also be constructed with 802.11 wireless links (just like in Fig. 1), which is called wireless distribution system (WDS). In WDS-based WLANs, one AP (called base AP) is connected to the wired link and one or more APs (called linked AP) can be linked with the base AP or other linked APs, which forms several topologies such as star or tree.[5]

\section{B. The Network Simulator 3}

NS3 project started in 2006 as a continuation of NS2. This simulation environment was developed from ground-up, using parts that was previously developed in NS2, to create a complete software solution. This environment is supplied in the form of libraries and examples of models, that help in creation of your own simulation model. Also in the same package by default there is a graphical visualiser PyVis and system for simulation recording and playing NetAnim. Because this environment is supplied in the form of libraries, it has great capabilities for advancements and modification, which allows to retrieve results of simulation in desired form. Also environment allows to retrieve raw data on each of the modules in the process of modeling. NS3 also supplies full code documentation and instructions on how to make new models and modules for NS3.[6]

\section{MODELING SYSTEM}

A. Network Design

This research is conducted specifically to find the best network model of WDS application in campus environment. The WDS network architecture is designed using three scenario models:

\section{WDS Bridge Point-to-point}

2. WDS Bridge Point-to-multipoint

\section{WDS Repeater}

Scenario testing is done by testing the effect of increasing the number of clients starting from 2, 4, 6 to 8 client. The measurement parameters taken are throughput, Packet Delivery Ratio (PDR) and delay.

B. WDS Network Scenario Model

Based on the initial observation of WDS topology scenario placement in campus environment, there are three WDS network models that are being implemented:

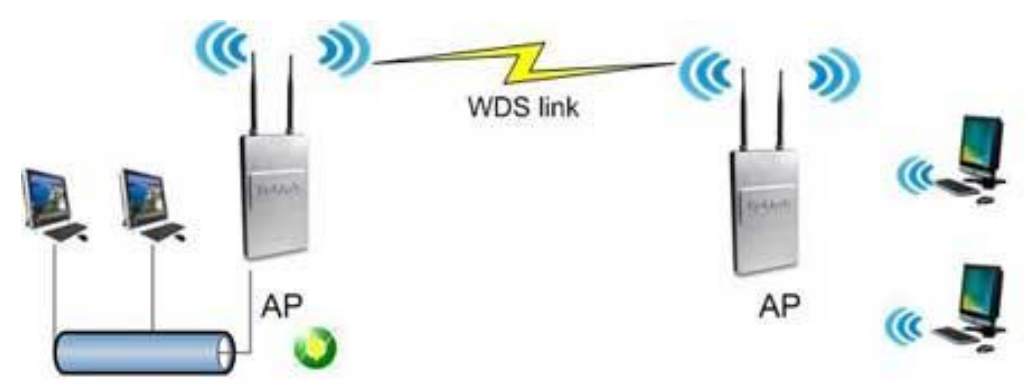

Figure 1. WDS Bridge Point-to-point

Figure 1. Above shows the topology scenario of WDS Bridge point-to-point. This scenario is done to see the effect of WDS link if it were connected between 2 Access Point directly. This simulation is done by increasing the number of clients. 


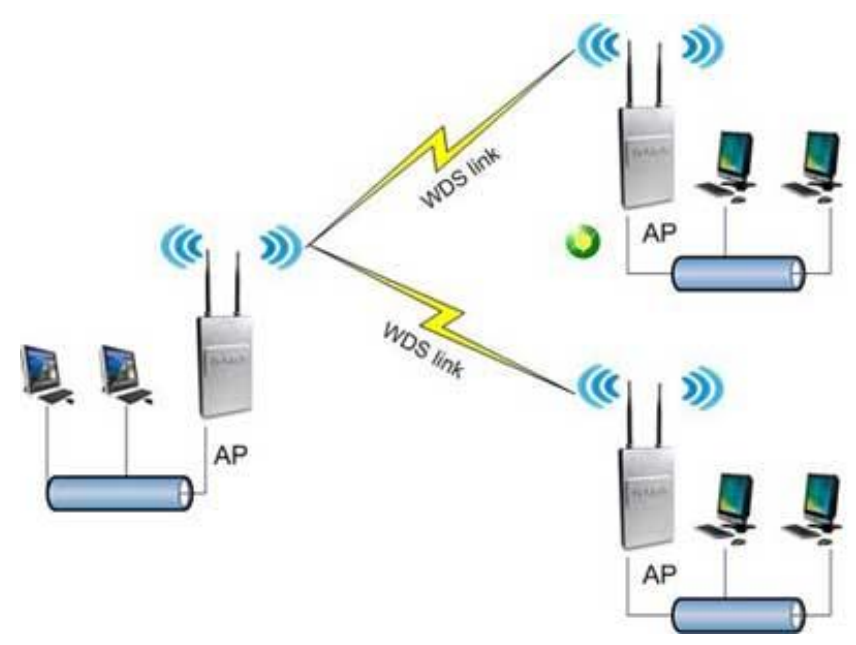

Figure 2. WDS Bridge Point-to-Multipoint

Figure 2. Above shows the topology scenario of WDS Bridge point-to-multipoint. This scenario is done to see the effect on QoS if the Access Point source handles 2 Access Point simultaneously using 2 pieces of WDS link. This simulation is done by increasing the number of clients from 2 to 8 clients.

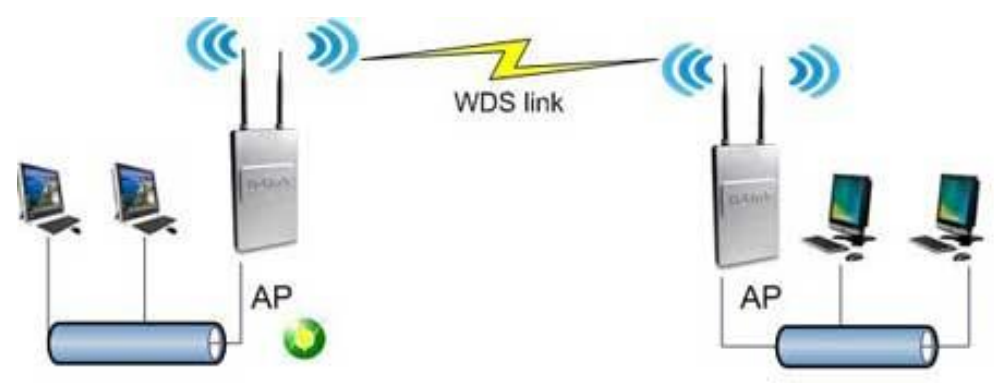

Figure 3. WDS Repeater

Figure 3. Above shows the topology scenario of WDS repeater where this scenario is performed to see the effect on QoS if at first the Access Point source is connected to the client through the Access Point repeater. This condition may occur when the Access Point that handles the client is too far away from its position with the source of Access Point. This simulation is done by increasing the number of clients from 2 to 8 clients.

The scenario is created using Network Simulator 3 (NS3) simulator. On each client will then be generated TCP traffic by targeting one of the APs that have connection to the internet. The following are the simulation parameters performed on each WDS scenario.

\begin{tabular}{|l|c|}
\hline \multicolumn{1}{|c|}{ Parameter } & Values \\
\hline Simulation Time & $60 \mathrm{~s}$ \\
\hline Number of client $(\mathrm{s})$ & $2,4,6,8$ \\
\hline Simulation area $(\mathrm{m})$ & $40 \times 40$ \\
\hline Transport Protocol & TCP \\
\hline
\end{tabular}

Each scenario will use the above simulation parameters by taking into account the output parameters of the simulation: throughput, PDR, and delay. The following is a topology overview built using NS3 for each WDS scenario.

The first simulated topology is the WDS bride point to point mode. In this simulation used two mikrotik which serves as access point. The number of users is start from 2, 4, 6 and 8 users to see the effect of the number of users on the parameters to be measured. In this configuration all access points can serve requests from users, but AP 1 must share bandwidth with AP 2. Configuration on NS-3 is shown in Figure 4. 


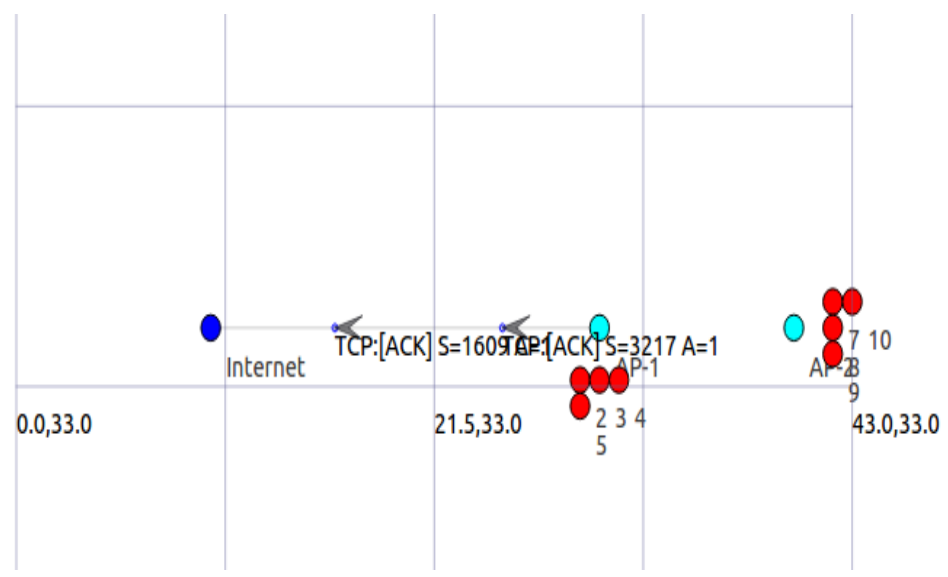

Figure 4. WDS bridge point-to-point

Unlike the previous configuration in point to multipoint configuration used three pieces of AP where AP1 serves as a bridge for AP2 and AP3. In this configuration (Figure 5) it appears that the access client is only served on AP2 and AP3. The number of users starts from 2, 46 and 8 users to see the effect of the number of users on the measured QOS parameters.

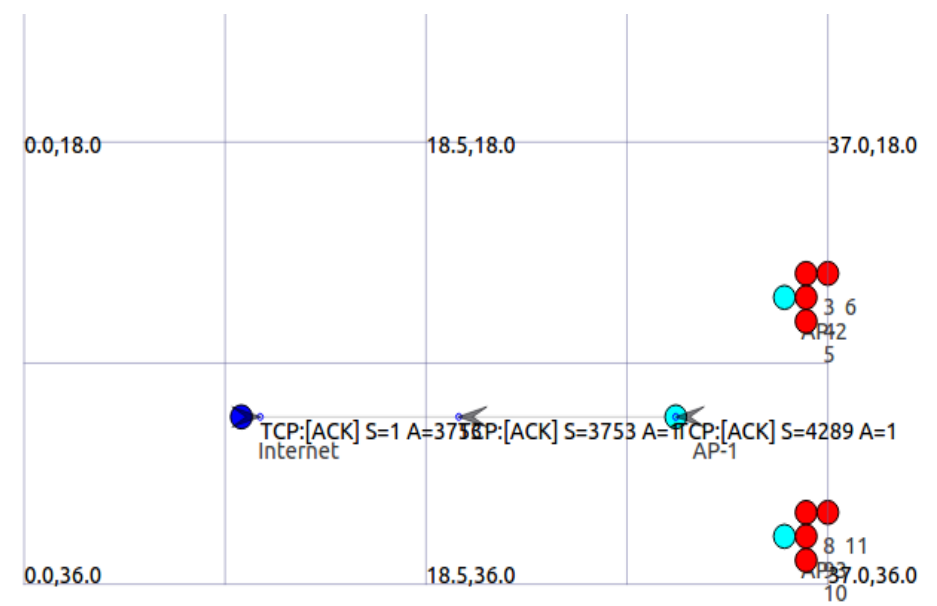

Figure 5. WDS bridge point-to-multipoint

In repeater mode (Figure 6), three APs are used where AP1 and AP2 serve as a bridge for AP3. User clients are only served on AP3. The repeater function here appears on AP2 located between AP1 and AP2. The number of users starts with 2, 46 and 8 users to see the effect of the number of users on the measured QOS parameters.

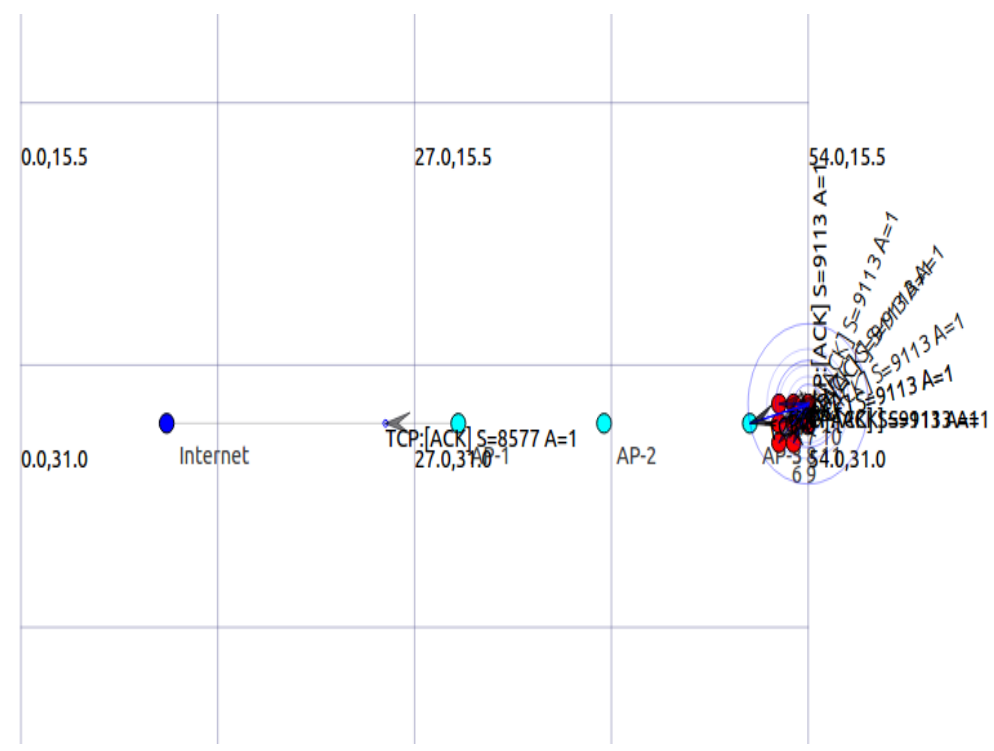

Figure 6. WDS repeater 


\section{SIMULATION AND ANALYSIS}

The results was obtained from the simulation using NS3 with simulation parameters according to the previous chapter for each simulation scenario. The types of data to be considered are throughput, PDR and delay.

\section{Increase Clients Scenario}

\section{a. Throughput Measurement}

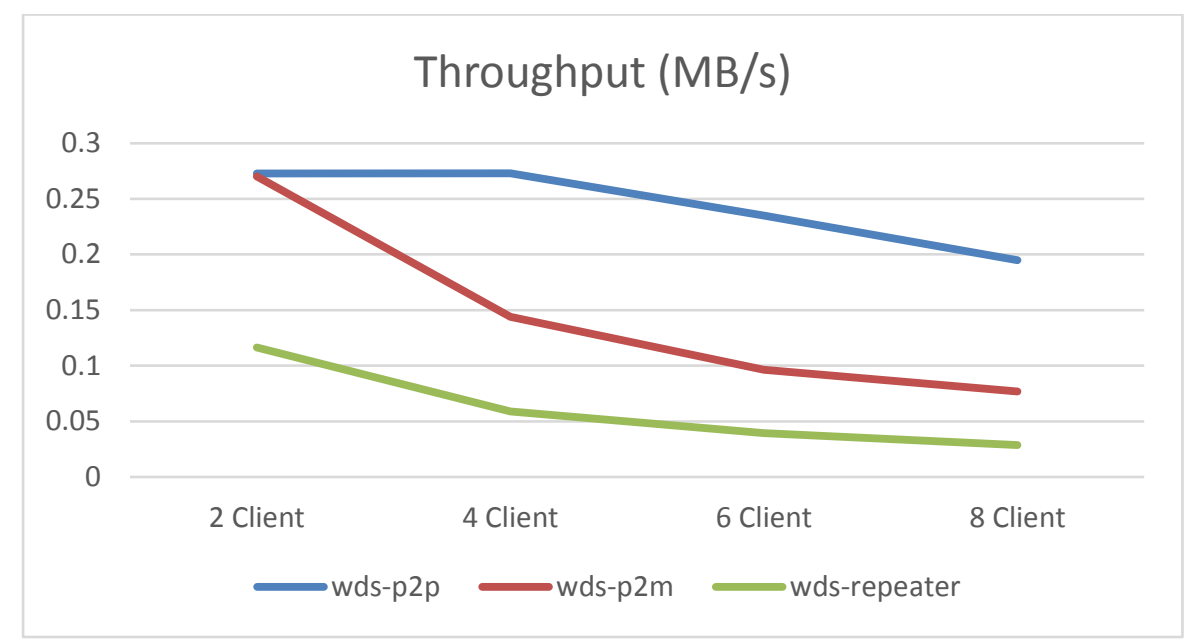

Figure 7: The effects of increase client towards throughput

The graphic shows that the more clients we have, the more throughput decreases. From the three scenarios performed it shows that the WDS point to point topology provides higher throughput results compared to the topology point to multipoint and repeater topology. This result is because the point to point topology does not pass through several WDS networks and directly connects with the intended AP directly so that succeeds in sending larger data.

\section{b. Packet Delivery Ratio (PDR) Measurement}

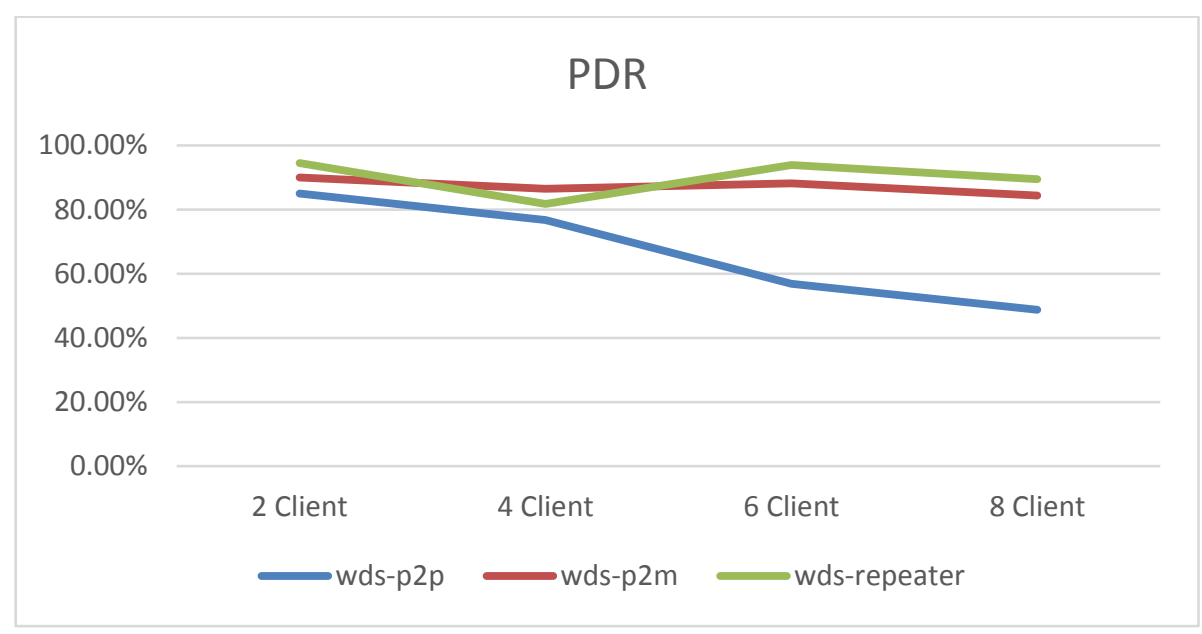

Figure 8: The effects of increase client towards PDR

PDR is affected by the throughput value in measuring the performance of WDS topology in packet data transfer. The higher resulting of PDR value gives a picture of PDR performance in sending packet data from various clients. PDR is measured by percentage of success while throughput is measured by unit per time. Based on the three topologies done seen that the increase number of clients impact the value of PDR decreased, this can happen because the link work process in the search path to the destination client.

\section{c. Average End to End Delay}




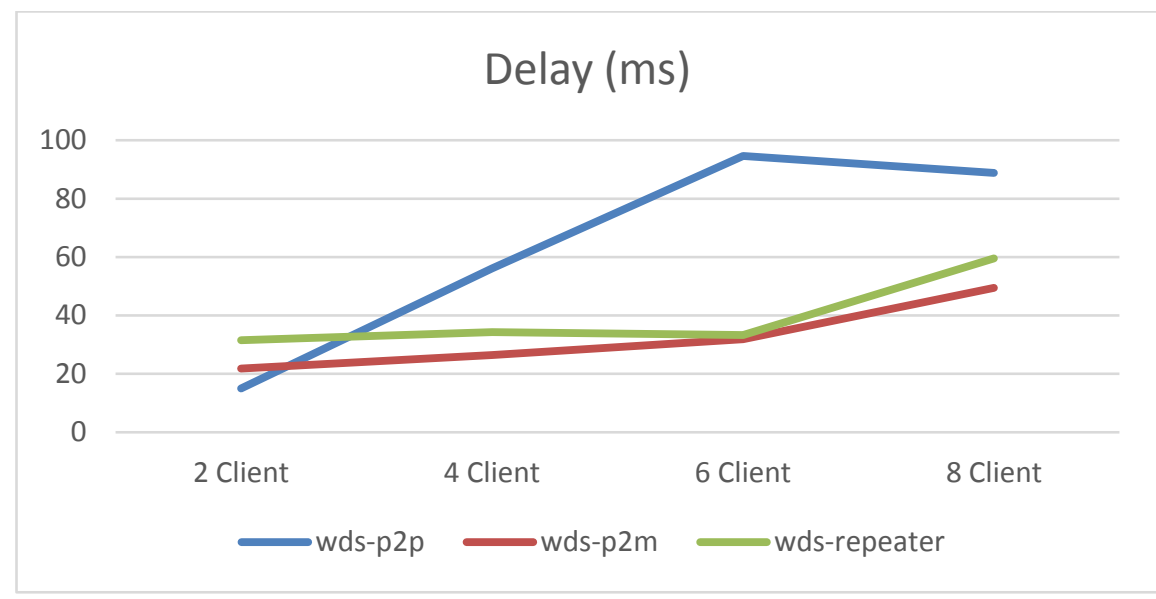

Figure 9: The effect of increase client towards delay

The next test is to measure the time a packet uses when sending data from one point to another or end-to-end delay. The time needed to find the route is one of the factors that affect the amount of time delay. This is because the source node must know in advance where the position of the destination node.

From the graph it can be concluded that the increasing number of WDS clients resulting in a higher delay. The WDS point to point topology delivers high delay but still below the ITU-T delay standard of 150ms. These results have an impact on the throughput of the WDS point to point topology that gives the best results. This suggests that topology point to point could guarantee more success in delivering data compared to delay. But for real-time services, this condition should be further investigated.

\section{Conclusion}

Legacy WLAN Station is usually associated with AP with the highest signal strength. After measuring and improving the placement of AP WDS in accordance with three topologies, three parameters of QoS are obtained through Throughput, PDR and Delay. From the increase client scenario for throughput parameter, it is found that the topology point to point gives better value but it generated higher delays. The delay generated on point to point is higher than other topologies. Meanwhile, the measurement results are not much different on the topology point to multipoint with repeater topology. The point to multipoint topology is slightly better than the repeater of the three QoS parameters. The result suggest that WDS is better at increasing the range of network coverage. On the other side, QoS results are less satisfactory that had been anticipated. Thus it can be concluded that WDS is not the best choice in many cases, especially on WLAN Based WDS.

\section{REFERENCES}

[1] IEEE, IEEE 802.11 : Part 11 : Wireless LAN Medium Access Control MAC and Physical Layer PHY specifications. IEEE, 1999.

[2] IEEE, IEEE 802.11b : Part 11 : Wireless LAN Medium Access Control MAC and Physical Layer PHY specifications: Higher-Speed Physical Layer Extension in the 2.4 GHz Band. IEEE, 2000.

[3] R. Tahar, A. Belghith, and R. Braham, "Performance evaluation of ieee 802.11 multi-interface based wireless distribution system (mi-wds)," in Computer Systems and Applications, 2009. AICCSA 2009. IEEE/ACS International Conference on, May 2009, pp. 764-770.

[4] R. Lara-Cueva, D. Benitez, and C. Fernandez "Performance Analysis of Wireless Network Modes in Conformance with IEEE 802.11b and WDS", in Asia-Pacific Conference on Computer Aided System Engineering (APCASE), 2015.

[5] J-P. Jeong, W-S. Lim, and Y-J. Suh, "Throughput Enhancement for WDS-based WLANs", in 22nd International Conference on Computer Communications and Networks (ICCCN), 2013.

[6] A. Bakhtin, A.Volkov, S. Muratchaev, V. Galenko, and A. Konoplev, " Development of MANET network model for space environment in NS3", in IEEE Conference of Russian Young Researchers in Electrical and Electronic Engineering (EIConRus), 2017.

[7] B. Zubairu, "Designing of Wireless Distribution System Network (WDSN) Model”, in Academic Journal of Interdisciplinary Studies, MCSER Publishing, Rome-Italy, Vol 2 No 7, September 2013.

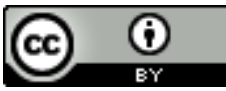

This work is licensed under a Creative Commons Attribution 4.0 International License.

DOI:10.24297/ijct.v16i5.6250

$6925 \mid$ P a g e
July, 2017

w w w. cirworld.com 